\title{
A small interfering RNA targeting vascular endothelial growth factor efficiently inhibits growth of VX2 cells and VX2 tumor model of hepatocellular carcinoma in rabbit by transarterial embolization-mediated siRNA delivery
}

This article was published in the following Dove Press journal:

Drug Design, Development and Therapy

24 March 2016

Number of times this article has been viewed

\begin{abstract}
Yu Zou, ${ }^{1,2}$ Chuan-Gen Guo, ${ }^{2}$ Zheng-Gang Yang, ${ }^{3}$ Jun-Hui Sun, ${ }^{4}$ Min-Ming Zhang, ${ }^{5}$ CaiYun $\mathrm{Fu}^{6,7}$

'Department of Radiology, Women's Hospital, School of Medicine, Zhejiang University, ${ }^{2}$ Department of Radiology, First Affiliated Hospital, School of Medicine, Zhejiang University, ${ }^{3}$ Institute of Infectious Diseases, First Affiliated Hospital, School of Medicine, Zhejiang University, ${ }^{4}$ Division of Hepatobiliary and Pancreatic Surgery, Department of Surgery, First Affiliated Hospital, School of Medicine, Zhejiang University, ${ }^{5}$ Department of Radiology, Second Affiliated Hospital, School of Medicine, Zhejiang University, ${ }^{6} \mathrm{College}$ of Life Sciences, Zhejiang Sci-Tech University, ${ }^{7}$ Key Laboratory of Cell-Based Drug and Applied Technology Development in Zhejiang Province, Hangzhou, People's Republic of China
\end{abstract}

Correspondence: Cai-Yun Fu College of Life Sciences, Zhejiang Sci-Tech University, Hangzhou 3100 18, People's Republic of China

Tel +86 57I 86843336

Email fucy03@126.com

Min-Ming Zhang

Department of Radiology, Second Affiliated Hospital, School of Medicine, Zhejiang University, Hangzhou 310003, People's

Republic of China

Tel +86 57| 873। 5255

Email zhangminming@zju.edu.cn
Introduction: Hepatocellular carcinoma is currently the second leading cause of cancer-related deaths worldwide with an increasing incidence.

Objective: The objective of this study is to investigate the effect of vascular endothelial growth factor small interfering RNA (VEGF-siRNA) on rabbit VX2 carcinoma cell viability in vitro and the effect of transarterial embolization (TAE)-mediated VEGF-siRNA delivery on the growth of rabbit VX2 liver-transplanted model in vivo.

Methods: Quantitative reverse transcription polymerase chain reaction, enzyme-linked immunosorbent assay, and Western blot technologies were used to detect the expression level of VEGF. TAE and computed tomography scan were used to deliver the VEGF-siRNA and detect the tumor volume in vivo, respectively. Microvessel density was detected by immunohistochemistry with CD34 antibody. A biochemical autoanalyzer was used to evaluate the hepatic and renal toxicity.

Results: The designed VEGF-siRNAs could effectively decrease the expression levels of VEGF mRNA and protein in vitro and in vivo. In vitro, the viability of rabbit VX2 carcinoma cells was reduced by $38.5 \% \pm 7.3 \%$ (VEGF-siRNA no 1 ) and $30.0 \% \pm 5.8 \%$ (VEGF-siRNA no 3 ) at 48 hours after transfection. Moreover, in rabbit VX2 liver-transplanted model, the growth ratios of tumors at 28 days after TAE-mediated siRNA delivery were $155.18 \% \pm 19.42 \%$ in the control group, $79.67 \% \pm 19.63 \%$ in the low-dose group, and $36.09 \% \pm 15.73 \%$ in the high-dose group, with significant differences among these three groups. Microvessel density dropped to $34.22 \pm 4.01$ and $22.63 \pm 4.07$ in the low-dose group and high-dose group, respectively, compared with the control group (57.88 \pm 5.67$)$, with significant differences among these three groups. Furthermore, inoculation of VX2 tumor into the liver itself at later stage induced significant increase in alanine aminotransferase and aspartate aminotransferase, indicating an obvious damage of liver functions, while treatment of VX2 tumor via TAE-mediated VEGF-siRNA had no toxicity to the livers and kidneys of rabbits, and VEGF-siRNA had the ability to protect liver damage induced by tumor growth.

Conclusion: This is the first study to demonstrate that targeting VEGF via TAE-mediated siRNA delivery may become a powerful new option for effective treatment of hepatocellular carcinoma in the clinic.

Keywords: hepatocellular carcinoma, VEGF-siRNAs, CT scan, microvessel density, toxicity to the livers and kidneys 


\section{Introduction}

Hepatocellular carcinoma (HCC) is currently the second leading cause of cancer-related deaths worldwide with an increasing incidence ${ }^{1}$ and is one of the most common hypervascular tumors with a poor prognosis owing to largely ineffective therapeutic options. ${ }^{2-4}$ The treatment options for patients with HCC currently include resection, transplantation, and percutaneous ablation, depending on tumor size and staging. ${ }^{1}$ The palliative treatments include transarterial chemoembolization (TACE), radioembolization, and systemic treatments. ${ }^{1}$ Although surgical resection is the first choice for patients with HCC with 5-year survival rates up to $70 \%,{ }^{5,6}$ it has been somewhat limited because $>80 \%$ of patients have liver cirrhosis. ${ }^{1}$ In addition, the growth sites of some HCC are near the first, second, and third hepatic portals, which are very hard for resection, resulting in a proportion of $<20 \%$ of clinical patients with $\mathrm{HCC}$ for resection. ${ }^{7,8}$ In HCC, $90 \%$ of blood supply is obtained from the hepatic artery and only $10 \%$ from the portal vein. ${ }^{9}$ In clinical patients with HCC, one reason for unfavorable prognosis is the high incidence of early microvascular invasion. ${ }^{10}$ Obviously, angiogenesis and the production of angiogenic factors are essential for tumor growth, invasion, and metastasis. ${ }^{11}$ Thus, TACE is the most common option used in the treatment of HCC in order to block the blood supply and induce tumor necrosis, without markedly affecting the hepatic blood supply, ${ }^{1}$ which provides a new approach and management for $\mathrm{HCC}$ treatment without the inconvenience of resection. ${ }^{12}$

However, in our clinical practice, the protective efficacy of TACE for patients with HCC is unsatisfactory, with a high incidence of recurrence and metastasis, inducing a high death incidence. One important reason for this kind of unsatisfactory outcome is the upregulated expression level of vascular endothelial growth factor (VEGF) induced by ischemia and anoxia after TACE operation. ${ }^{13-19}$ Moreover, VEGF is known to be central to promote tumor angiogenesis, and VEGF has been shown to increase vascular permeability, which is 50,000 times greater than the effect of histamine. ${ }^{20,21}$ Thus, the expression level of VEGF is correlated with the proliferation, metastasis, and the construction level of blood vessels, which directly indicates the proliferative rate and metastasis tendency of tumors. ${ }^{22}$ In order to reduce the incidence of recurrence and metastasis after TACE operation for patients with $\mathrm{HCC}$, it is essential to simultaneously block the signal transduction of VEGF and to reduce the expression level of VEGF, which can prevent neovascular formation and improve the patients' survival rate. In the clinic, it is urgent and important to combine the TACE technology and targeting blocking technology of VEGF expression to improve the therapeutic effect of TACE.

RNA interference is an evolutionarily conserved surveillance mechanism through which 21- and 22-nucleotide small interfering RNAs (siRNAs) induce sequence-specific post-transcriptional gene silencing in many organisms. ${ }^{23-25}$ Using RNA interference technology, previous studies showed that siRNAs targeting VEGF inhibited gastric cancer cell growth ${ }^{26}$ and gastric cancer growth in a nude mouse model of subcutaneous xenografts, ${ }^{27}$ inhibited the growth of malignant melanoma, ${ }^{28}$ and inhibited the tumor growth and tumor angiogenesis in orthotopic tumors in mice through intraperitoneal application of siRNA-VEGF. ${ }^{29} \mathrm{Up}$ to now, the most commonly used method for siRNAs delivery was tumor local injection in subcutaneous xenografts with the disadvantages of somewhat diverse delivery method from human clinical applications. Recently, some studies have reported the drug delivery method for siRNA by intravenous injection, with the disadvantages of poor targeting to tumors and high drug toxicity, which restricts its application in the clinic..$^{30-32}$ In order to overcome the aforementioned disadvantages, in this study, we plan to use the transarterial embolization (TAE) technology to deliver the siRNAs for the first time and to evaluate the effect of VEGF knockdown on tumor growth of HCC in vivo via interventional treatment of HCC in clinical application with two aspects of advantages. One advantage is the fact that the unique affinity and avocation of HCC to lipiodol to improve the targeting property. 33,34 Another advantage is that lipiodol may have the property to wrap up the siRNAs as an emulsifying agent to protect the siRNAs from degradation by the substances in blood.

\section{Materials and methods Cell line and animals}

Rabbit VX2 carcinoma cell line used in this study was preserved in our laboratory, which was bought from Chinese Academy of Medical Sciences. The adult New Zealand White rabbits were provided by Zhejiang Academy of Medical Sciences, Hangzhou, People's Republic of China. All experiments were approved by the animal research committee of Zhejiang University. Every effort was made to minimize the numbers and any suffering of the animals used in the following experiments.

\section{Preparation of siRNAs}

Three siRNAs targeting rabbit VEGF and one scrambled siRNA (used for a negative control) with the sense and antisense sequences were designed according to the rabbit 
Table I Design of the potential three siRNA sequences targeting VEGF gene and one scrambled siRNA (scr-siRNA, used for a negative control), as well as their corresponding targeted sites

\begin{tabular}{llll}
\hline siRNA ID & Sense sequences & Antisense sequences & VEGF target sites \\
\hline VEGF-siRNAI & UCGAGACCUUGGUGGACAUdTdT & AUGUCCACCAAGGUCUCGAdTdT & nt26-nt44 \\
VEGF-siRNA2 & GGAGUACCCUGAUGAGAUCdTdT & GAUCUCAUCAGGGUACUCCdTdT & nt5I-nt69 \\
VEGF-siRNA3 & UGAUGAAAGCCUGGAGUGCdTdT & GCACUCCAGGCUUUCAUCAdTdT & $\mathrm{nt}$ 126-ntI44 \\
scr-siRNA & UUCUCCGAACGUGUCACGUdTdT & ACGUGACACGUUCGGAGAAdTdT & - \\
\hline
\end{tabular}

Abbreviations: siRNA, small interfering RNA; VEGF, vascular endothelial growth factor.

VEGF messenger RNA (mRNA) complete sequence for all the VEGF isoform in NCBI. The targeting sequence and its location of each siRNA in rabbit VEGF cDNA were summarized in Table 1. The selected siRNA sequences were blast searched against expressed sequence tag libraries to ensure that only a single gene is targeted. All siRNAs were synthesized by Biomics Biotechnologies Co., Ltd. (Jiangsu, People's Republic of China) using 2'-bis (acetoxyethoxy)-methyl ether protection chemistry as mentioned in previous report. ${ }^{35}$

\section{Cell culture conditions and transfection of siRNAs}

The VX2 cells were cultured in Dulbecco's Modified Eagle's Medium (Thermo Fisher Scientific, Waltham, MA, USA) supplemented with $10 \%$ heat-inactivated fetal bovine serum (Gibco BRL; Thermo Fisher Scientific) at $37^{\circ} \mathrm{C}$ in a humidified atmosphere at $5 \% \mathrm{CO}_{2}$. Cells were seeded in a 12 -well plate with a density of $5 \times 10^{5}$ cells/well. After 24 hours and $70 \%-80 \%$ confluence, the cells were transfected with respective VEGF siRNAs $(50 \mathrm{nM})$ and scr-siRNA (50 $\mathrm{nM})$ in serum-free medium using Lipofectamin $2000^{\mathrm{TM}}$ (Thermo Fisher Scientific) according to manufacturer's instructions. After incubation for 6 hours at $37^{\circ} \mathrm{C}$, the medium in each well was then replaced with Dulbecco's Modified Eagle's Medium, with $10 \%$ heat-inactivated fetal bovine serum for another 48 hours. The conditioned medium in each well was collected for enzyme-linked immunosorbent assay (ELISA), and the cells in each well were collected for quantitative reverse transcription polymerase chain reaction (RT-qPCR) assay. The FAM-scr-siRNA was used to optimize the proportion of siRNA and Lipofectamin 2000 using an inverted fluorescence microscope (TE2000-U; Nikon Corporation, Tokyo, Japan) as described previously. ${ }^{36}$

\section{VEGF ELISA}

Quantikine rabbit VEGF Immunoassay kit (R\&D Systems, Inc., Minneapolis, MN, USA) was used to detect the secretion of VEGF into the cell culture supernatant according to manufacturer's instructions. Inhibitory rate of secreted
VEGF $(\%)=([\mathrm{VEGF}$ content in nontransfected group VEGF content in transfected group]/VEGF content in nontransfected group) $\times 100$.

\section{RT-qPCR analysis for VEGF gene expression}

The VX2 cells for RNA extraction collected above were washed with phosphate-buffered saline for three times. RNA was isolated using TRIzol Reagent (Thermo Fisher Scientific) according to the manufacturer's instructions. In all experiments, $2 \mu \mathrm{g}$ of total RNA was reverse transcribed with M-MLV reverse transcriptase (TIANGEN) into cDNA in a final volume of $50 \mu \mathrm{L}$ as mentioned in our previous report. ${ }^{37}$ In order to eliminate the variations in each group, glyceraldehyde 3-phosphate dehydrogenase (GAPDH) was used as an endogenous internal control gene. An ABI7500 real-time PCR detection system apparatus was used to perform the quantitative expression study. The PCR forward primer and reverse primer for VEGF were 5'-GAGACCTTGGTGGACATC-3' and 5'-CCAGGCTTTCATCATTGC-3', respectively. The PCR forward primer and reverse primer for GAPDH were 5'-CAAGATTGTCAGCAACGCAT-3' and 5'-GTCTTCTGGGTGGCAGTGAT-3', respectively. The molecular sizes of amplification products for VEGF and GAPDH were 112 bp and 129 bp, respectively. The specificity of the PCR reactions was detected by size verification of the amplicon in a conventional $2 \%$ (wt/vol) agarose gels containing $0.05 \mathrm{mg} / 100 \mathrm{~mL}$ ethidium bromide. The $2^{-\Delta \Delta \mathrm{Ct}}$ method was used to calculate the relative expression level of VEGF gene as mentioned in our previous report. ${ }^{37}$ All reactions were run in triplicate and independently repeated at least three times.

\section{Cell viability assay}

VX2 cells were harvested during the logarithmic growth phase and seeded in a 96-well plate at a density of $5 \times 10^{3}$ cells/well. Then the cells in each well were transfected with siRNAs using the method mentioned earlier. After 48 hours incubation, $10 \mu \mathrm{L}$ 3-(4,5-dimethylthiazol-2-yl)-2, 
5-diphenyltetrazolium bromide solution (Sigma-Aldrich Co., St Louis, MO, USA; $5 \mathrm{mg} / \mathrm{mL}$ ) was added to each well and incubated at $37^{\circ} \mathrm{C}$ in $5 \% \mathrm{CO}_{2}$ for 4 hours. Dimethyl sulfoxide (Sigma-Aldrich Co.) at the volume of $150 \mu \mathrm{L}$ was used to dissolve the formazan crystals, and the absorbance was measured at $490 \mathrm{~nm}$ using microplate reader (Varioscan Flash; Thermo Fisher Scientific) as mentioned in our previous report. ${ }^{38}$ Proliferative inhibitory rate $(\%)=\left(\left[\mathrm{OD}_{490}\right.\right.$ value in nontransfected group - $\mathrm{OD}_{490}$ value in transfected group]/ $\mathrm{OD}_{490}$ value in nontransfected group) $\times 100$.

\section{Tumor implantation and experimental design}

A total of 30 rabbits $(2.0-2.5 \mathrm{~kg})$ were used for this experiment. The VX2 tumor model was induced and maintained by means of successive transplantation into the hind limb of a carrier rabbit. The donor tumor was excised and minced, and tumor cells $(0.1 \mathrm{~mL})$ were directly implanted into the subcapsular parenchyma of the left hepatic lobe after making a midline subxiphoid abdominal incision. The rabbits were maintained for 28 days after tumor implantation when the tumors were anticipated to be $15-50 \mathrm{~mm}$ in diameter.

One day prior to TAE, a liver computed tomography (CT) scan was performed (Philips Brilliance 64; Philips Medical Systems, Best, the Netherlands) with the animals in the supine position. The CT parameters included $3 \mathrm{~mm}$ collimation, 1.5 pitch, and a $1 \mathrm{~mm}$ reconstruction interval with $30 \%$ overlap, $120 \mathrm{kVp}$, and $120 \mathrm{~mA}$. Triphasic CT scans were performed in the nonenhanced, arterial, and portal venous phases with an injection of $13 \mathrm{~mL}$ of nonionic contrast material (Ultravist; Schering, Berlin, Germany), at a rate of $0.5 \mathrm{~mL} / \mathrm{s}$, through the auricular vein.

On the CT scan, the sizes of the tumors were measured by two authors working in consensus. The tumor volume $(V)$ was calculated according to the following equation: $V=a \times b^{2} / 2$, where " $a$ " is the longest diameter and " $b$ " is the shortest diameter of the tumor. The animals with similar size tumors were distributed evenly to each group on the basis of the calculated tumor volume because the results of antitumor treatment can be influenced by the tumor volume. ${ }^{39,40}$ Thirty rabbits were divided into three groups ( $\mathrm{n}=10$ for each group) and treated with TAE with high-dose siRNA $(5 \mathrm{mg} / \mathrm{kg})$ plus Lipofectamin $2000(0.76 \mathrm{~mL} / \mathrm{kg})$ and $0.2 \mathrm{~mL}$ lipiodol (Andre Guerbet, Aulnay-sous-Bois, France), low-dose siRNA (1 mg/kg) plus Lipofectamin $2000(0.15 \mathrm{~mL} / \mathrm{kg})$, and $0.2 \mathrm{~mL}$ lipiodol, or control (2 mL normal saline and $0.2 \mathrm{~mL}$ lipiodol only), in a total volume of $2.2 \mathrm{~mL}$ for each group. The mixture was shaken with a vortex machine (WBZ-3222;
Shibei Bioscience, Shanghai, People's Republic of China) for 1 minute at room temperature to make into an emulsifying agent. The mixed solution was immediately used for TAE.

\section{Transarterial embolization}

Four weeks after implantation of VX2 tumor in the liver, TAE was performed under fluoroscopic guidance by one interventional radiologist as shown in Figure 1. General anesthesia was induced, and the right femoral artery was exposed. A 5-Fr dilator, as a substitute for an arterial sheath, was inserted into the right femoral artery. With a 3 Fr microcatheter (Progreat; Terumo, Tokyo, Japan), celiac angiography was performed to identify the hepatic arterial anatomy and the feeder artery of the tumor. The left hepatic artery, which exclusively supplied blood flow to the tumor, was selectively catheterized via the common hepatic artery. After positioning the catheter within the left hepatic artery, a diagnostic angiogram was obtained. The prepared emulsion with a fixed volume was injected carefully to avoid efflux of the embolic materials out of the artery. The catheter was then removed, and the femoral artery was ligated.

\section{Assessment of tumor growth ratio}

Twenty-eight days after TAE, follow-up CT examination was performed to identify deposit of lipiodol and to measure tumor growth ratio (GR). The GR was calculated by comparing the tumor volumes obtained before $(\mathrm{Vb})$ and 28 days after

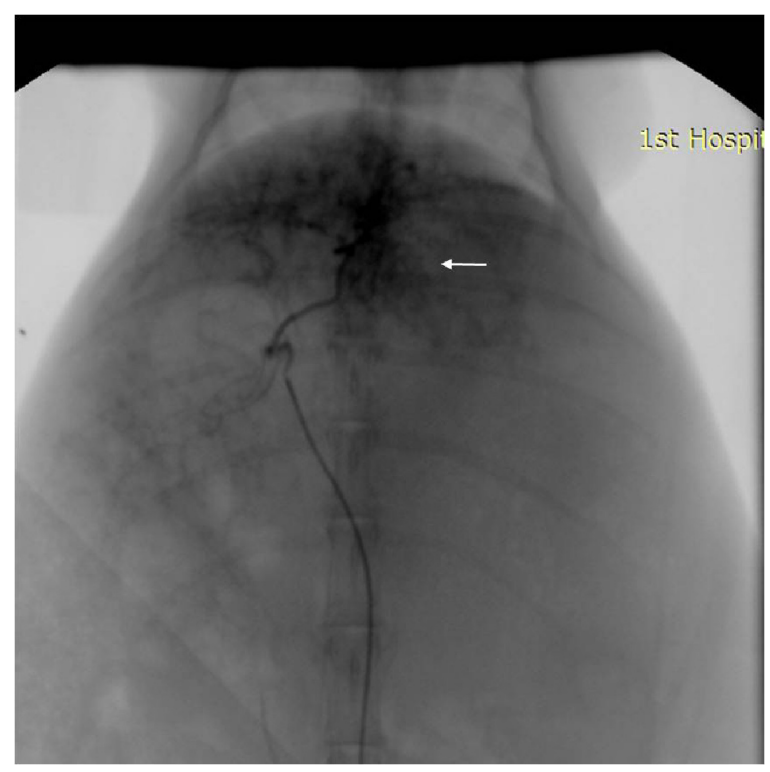

Figure I A diagnostic angiogram obtained after selectively positioning the $3 \mathrm{Fr}$ catheter within the left hepatic artery.

Note: White arrow indicates the hypervascular tumor staining in the left hepatic lobe. 
(V28) treatment according to the following formula: GR = $(\mathrm{V} 28 / \mathrm{Vb}-1) \times 100 \%$.

\section{Histologic and microvessel staining analysis}

The rabbits were euthanized 28 days after TAE, and their livers were explanted for histopathologic examination, RT-qPCR, and Western blot analysis. The liver was sliced at $5 \mu \mathrm{m}$ intervals in the axial plane, approximating the CT slice plane. The liver containing tumor that was easily identifiable was sliced in a similar fashion and then embedded for histopathologic examination. Vascularization and immunohistochemical staining were performed on the specimens with anti-CD34 monoclonal antibody according to the avidin-biotin peroxidase complex technique for evaluation. The vessel counting was performed as we described previously. ${ }^{41}$ In brief, the area of highest neovascularization was first identified by imaging of the tumor sections at low power $(\times 40$ and $\times 100)$. This area was then located subjectively, and individual microvessels were counted on a 200-power field in five to ten areas with an ocular graticule (area, $0.25 \mathrm{~mm}^{2}$ ) to enhance the precision. The mean counts in these five to ten areas were recorded. Microvessel density (MVD) counts were then determined by one pathologist without the knowledge of CT findings.

\section{RT-qPCR analysis for VEGF gene expression and Western blot analysis for VEGF protein expression in rabbits' livers}

The RT-qPCR analysis was performed as described previously.

Total proteins were separated by sodium dodecyl sulfate polyacrylamide gel electrophoresis on an $8 \%-10 \%$ gel. After electrophoresis, gels were transferred onto $0.45 \mu \mathrm{m}$ polyvinylidene difluoride membrane (EMD Millipore, Billerica, MA, USA) and blocked with $2 \%$ bovine serum albumin for 2 hours at room temperature. The membranes were incubated with the VEGF (Abcam) primary antibodies at $4{ }^{\circ} \mathrm{C}$ overnight. Secondary antibody is horseradish peroxidase-conjugated anti-rabbit (Jackson ImmunoResearch Laboratories, Inc., West Grove, PA, USA) for 2 hours before detecting by chemofluorescence detection kit (Amersham, Piscataway, NJ, USA) according to the manufacturer's instructions. GAPDH was used as a control.

\section{Assessment of hepatic and renal toxicity}

Peripheral blood was drawn from the auricular vein in all the rabbits at 1 day before, 7 days, and 28 days after TAE. The plasma aspartate aminotransferase (AST), alanine aminotransferase (ALT), blood urea nitrogen (BUN), and creatinine $(\mathrm{Cr})$ levels were measured with a biochemical autoanalyzer (TBA-200FR; Toshiba, Tokyo, Japan).

\section{Statistical analysis}

Each experiment was repeated at least three independent times. Data were expressed as mean \pm standard error of the mean (SEM). Comparisons of mean values were performed using one-way analysis of variance followed by the Dunnett's test for post hoc multiple comparisons. A value of $P<0.05$ was considered significant.

\section{Results siRNA transfection optimization}

The FAM-scr-siRNA (fluorescein-labeled siRNA) with the same sequence of scr-siRNA (Table 1) was used for siRNA protocol optimization to check for the transduction efficiency, as mentioned in a previous report. ${ }^{42}$ To test this, 12-well plates with VX2 cells were transduced with varied doses of Lipofectamin $2000(0 \mu \mathrm{L}, 0.5 \mu \mathrm{L}, 1 \mu \mathrm{L}$, and $1.5 \mu \mathrm{L}$, respectively) and FAM-scr-siRNA (10 nM, $20 \mathrm{nM}$, and $50 \mathrm{nM}$, respectively), as shown in Figure $\mathrm{S} 1$. Our results showed that the optimized transduction condition was $0.5 \mu \mathrm{L}$ of Lipofectamin 2000 and $50 \mathrm{nM}$ of FAM-scr-siRNA, which was optimal lipid transfection concentration with lower cell toxicity and higher transduction efficiency (Figure S1). So we used the optimal transfection condition for further study.

\section{siRNA efficacy analysis in vitro and in vivo}

In addition to lipid and siRNA optimization, it is also important that the assay can distinguish between siRNAs with different efficacies against a respective target. To test for VEGF siRNA target-specific efficacy, a negative control siRNA (scr-siRNA) was analyzed to provide a benchmark for off-target effects on VEGF knockdown (Table 1). In addition, we examined three VEGF siRNAs to target rabbit VEGF as described in Table 1. To determine whether the optimized transfection condition in VX2 cells mentioned previously also produced robust reduction in endogenous VEGF mRNA and protein via three technologies VEGF siRNAs, RT-qPCR, and ELISA, which were used to detect VEGF expression levels in vitro. Our results showed that the VEGF mRNA was significantly knocked out by $\sim 60 \%$ in the siRNA no 1 and no 3 group quantified using RT-qPCR technology and normalized to a nontransfected group (Figure 2). Moreover, of the three siRNAs synthesized, VEGF siRNA no 1 and no 3 also potently suppressed the synthesis and 

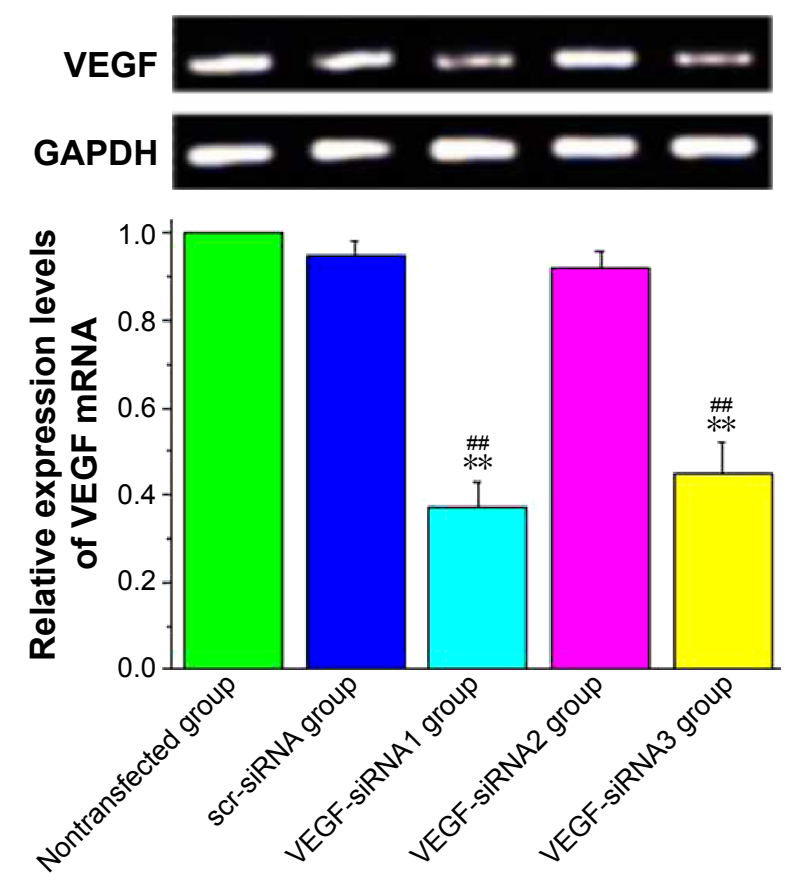

Figure 2 Quantitative determination of expression levels of VEGF mRNA in VX2 cells treated by three VEGF siRNAs and scr-siRNA at 48 hours by RT-qPCR assay. Notes: The VEGF mRNA levels were normalized to mRNA levels in nontransfected group (after internal GAPDH normalization). The error bars were standard error mean of at least three independent measurements. Representative RT-qPCR products were visualized after electrophoresis in I\% agarose gels stained with ethidium as shown on the upper portion. Significantly different from the nontransfected group: $* * P<0.01$; significantly different from the scr-siRNA group: ${ }^{\#} P<0.01$.

Abbreviations: GAPDH, glyceraldehyde 3-phosphate dehydrogenase; mRNA, messenger RNA; VEGF, vascular endothelial growth factor; siRNA, small interfering RNA; RT-qPCR, quantitative reverse transcription polymerase chain reaction.

secretion of VEGF protein in VX2 cells after transfection with Lipofectamin 2000, while the scr-siRNA showed no effect (Table 2). ELISA results for VEGF revealed that the inhibitory rates of secreted VEGF by VEGF siRNA no 1 and no 3 were $58.1 \% \pm 7.3 \%$ and $51.9 \% \pm 7.9 \%$, respectively (Table 2 ), which is coincident with the inhibitory rates at the mRNA level (Figure 2). Furthermore, we also detected the mRNA and protein expression levels of livers of rabbits at 28 days

Table 2 Comparison of VEGF expression levels in VX2 cells treated by three VEGF siRNAs and scr-siRNA at 48 hours by ELISA assay

\begin{tabular}{lll}
\hline Group & VEGF $(\mathrm{pg} / \mathrm{mL})$ & $\begin{array}{l}\text { Inhibitory rate } \\
\text { of secreted VEGF (\%) }\end{array}$ \\
\hline Nontransfected & $373.62 \pm 34.91$ & 0 \\
scr-siRNA & $361.41 \pm 31.04$ & $3.2 \pm 0.7$ \\
VEGF-siRNAI & $156.76 \pm 33.10 * * *$ & $58.1 \pm 7.3^{* *}$ \\
VEGF-siRNA2 & $357.54 \pm 30.20$ & $7.3 \pm 4.2$ \\
VEGF-siRNA3 & $179.20 \pm 29.36 * * *$ & $51.9 \pm 7.9 * *$ \\
\hline
\end{tabular}

Notes: Data were expressed as mean \pm standard error of the mean. $* P<0.0$ I, compared with nontransfected group. $* * P<0.01$, compared with scr-siRNA group. Abbreviations: VEGF, vascular endothelial growth factor; siRNA, small interfering RNA; ELISA, enzyme-linked immunosorbent assay. after TAE-mediated VEGF siRNA (no 1) in each group. As shown in Figure 3, the expression levels of VEGF mRNA (Figure 3A) and VEGF protein (Figure 3B) were significantly decreased in low-dose group ( $1 \mathrm{mg} / \mathrm{kg}$ ) and high-dose group (5 mg/kg), compared with corresponding control group.

\section{The viability inhibitory effect of VEGF siRNAs on VX2 cells}

To further investigate whether the VEGF siRNAs could effectively inhibit the viability of VX2 cells, the 3-(4,5dimethylthiazol-2-yl)-2,5-diphenyltetrazolium bromide assay was used to detect the viability inhibitory rate. After transfection of VEGF siRNAs $(50 \mathrm{nM})$ with Lipofectamin $2000(0.5 \mu \mathrm{L})$ for 48 hours, our results showed the viability inhibitory rate of VEGF siRNA no 1 and no 3 to VX2 cells were $38.5 \% \pm 7.3 \%$ and $30.0 \% \pm 5.8 \%$, respectively, while the scr-siRNA has no marked effect (Table 3 ). On the basis of these results, we selected VEGF siRNA no 1 as the most highly functional siRNA for further investigation on VX2 tumor model in vivo for HCC.

\section{TAE operation}

In all 30 rabbits, catheterization of the left hepatic artery was successful. Figure 1 showed a diagnostic angiogram obtained after selectively positioning the 3 Fr catheter within the left hepatic artery, as well as the hypervascular tumor staining in the left hepatic lobe. The 2-minute infusion (TAE procedure) and 1-hour operation with a total $2.2 \mathrm{~mL}$ of $1 \mathrm{mg} / \mathrm{kg}$ (lowdose group) and $5 \mathrm{mg} / \mathrm{kg}$ (high-dose group) VEGF siRNA no 1 were well-tolerated. All rabbits recovered without complications.

\section{Effect of VEGF siRNA on the GR of HCC}

Four weeks after inoculation of VX2 tumor into livers of rabbits, the CT scan technology was used to detect the liver tumor size at 1 day before TAE operation and at 28 days after TAE operation, respectively. Our results showed that the liver VX2 tumors were increased obviously in the control group but decreased significantly in the low-dose group and high-dose group of VEGF siRNA combined with lipiodol and Lipofectamin 2000 (Figure 4A). At the meantime, it was obvious that lipiodol was accumulated at the margins of the liver tumor and that there was obvious necrosis in the (middle) center of tumor obtained from the results of the contrast-enhanced CT image, as shown in Figure 4A. The statistical results showed that the GRs of tumors at 28 days after TAE operation were $155.18 \% \pm 19.42 \%$ in the control group, while the GRs in the low-dose group and high-dose group 

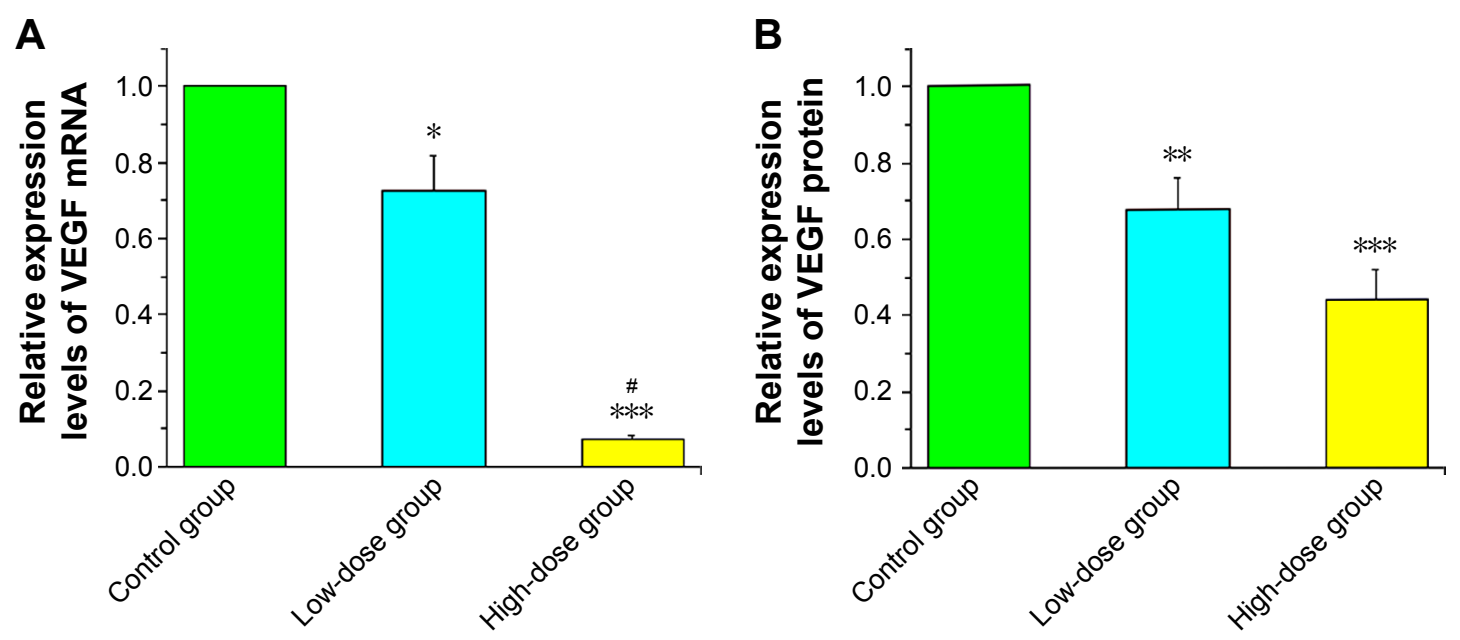

Figure 3 The expression levels of VEGF at 28 days after TAE-mediated VEGF siRNA in vivo.

Notes: (A) Relative expression levels of VEGF mRNA in each group. (B) Relative expression levels of VEGF protein in each group. $* P<0.05$, $* * P<0.0 \mathrm{I}$, and $* * * P<0.00 \mathrm{I}$, compared with each corresponding control group; ${ }^{\#}<0.05$, compared with low-dose group.

Abbreviations: VEGF, vascular endothelial growth factor; TAE, transarterial embolization; siRNA, small interfering RNA.

were $79.67 \% \pm 19.63 \%$ and $36.09 \% \pm 15.73 \%$, respectively (Figure 4B). There were significant differences among these three groups (Figure 4B).

\section{Effect of VEGF siRNA on MVDs}

The liver was taken out at 28 days after the CT scan, and the immunohistochemical technology was used to detect the MVDs of HCC using the CD34 monoclonal antibody (Figure 5A). Compared with the control group (57.88 \pm 5.67 of MVDs), the MVDs were $34.22 \pm 4.01$ and 22.63 \pm 4.07 in the low-dose group and high-dose group, respectively (Figure 5A and 5B). The MVDs in these three groups showed significant differences (Figure 5B).

\section{Toxicity evaluation of VEGF siRNA in livers and kidneys of rabbits}

The peripheral bloods of rabbits in each group were taken at 1 day before TAE operation, 7 days, and 28 days after TAE operation, respectively. Then we tested the levels of BUN, Cr,

Table 3 The viability inhibitory effect of VEGF siRNAs on VX2 cells at 48 hours by MTT assay

\begin{tabular}{lll}
\hline Group & OD $_{490}$ value & $\begin{array}{l}\text { Viability inhibitory } \\
\text { rate (\%) }\end{array}$ \\
\hline Nontransfected & $0.59 \pm 0.08$ & 0 \\
scr-siRNA & $0.51 \pm 0.06$ & $12.8 \pm 3.5$ \\
VEGF-siRNAI & $0.36 \pm 0.04^{* * *}$ & $38.5 \pm 7.3^{* *}$ \\
VEGF-siRNA2 & $0.50 \pm 0.07$ & $15.8 \pm 5.1$ \\
VEGF-siRNA3 & $0.4 I \pm 0.02^{* * *}$ & $30.0 \pm 5.8^{* *}$ \\
\hline
\end{tabular}

Notes: $* * * P<0.001$, compared with nontransfected group. $* * P<0.01$, compared with scr-siRNA group. Data is expressed as mean \pm standard error of the mean.

Abbreviations: VEGF, vascular endothelial growth factor; siRNA, small interfering RNA;MTT,3-(4,5-dimethylthiazol-2-yl)-2,5-diphenyltetrazoliumbromide;OD, optical density.
ALT, and AST in these peripheral blood samples. Our results showed that the levels of BUN and $\mathrm{Cr}$ were hardly changed in each group and each time point we tested (Figure 6A and B). The levels of ALT and AST were not marked changed at 1 day before TAE operation and 7 days after TAE operation in each group, while at 28 days after TAE operation, the levels of ALT and AST were increased significantly in the control group compared with the low-dose group and highdose group, respectively (Figure 6C and D).

\section{Discussion}

Treatment of patients with $\mathrm{HCC}$ is a big challenge in clinical practice based on the fact that patients with HCC need multidisciplinary clinical management and that selection of tailored treatments according to disease stage, patient age, and comorbidities. ${ }^{1} \mathrm{HCC}$ is a hypervascular tumor and highly resistant to currently available chemotherapeutic drugs. ${ }^{43}$ Therefore, strategies that reduce angiogenesis and vascular invasion may lead to beneficial outcomes for patients with HCC, especially in advanced stage tumors. ${ }^{44}$ siRNA is a powerful tool for controlling cellular processes of gene silencing at post-transcriptional level due to its high sequence-specific inhibition efficiency. ${ }^{45}$ In this study, we demonstrated that the VEGF siRNAs designed by ourselves have the ability to effectively knockdown VEGF expression at both mRNA and protein levels in vitro and in vivo. Meanwhile, VEGF siRNAs have the ability to inhibit the viability of rabbit VX2 carcinoma cells in vitro for the first time, which is coincident with previous reports that VEGF siRNAs could inhibit the proliferation of several kinds of tumor cell lines. ${ }^{46-50}$ 


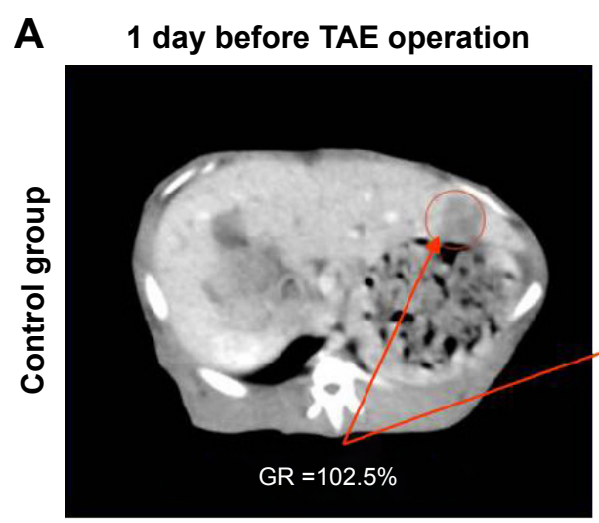

\section{8 days before TAE operation}
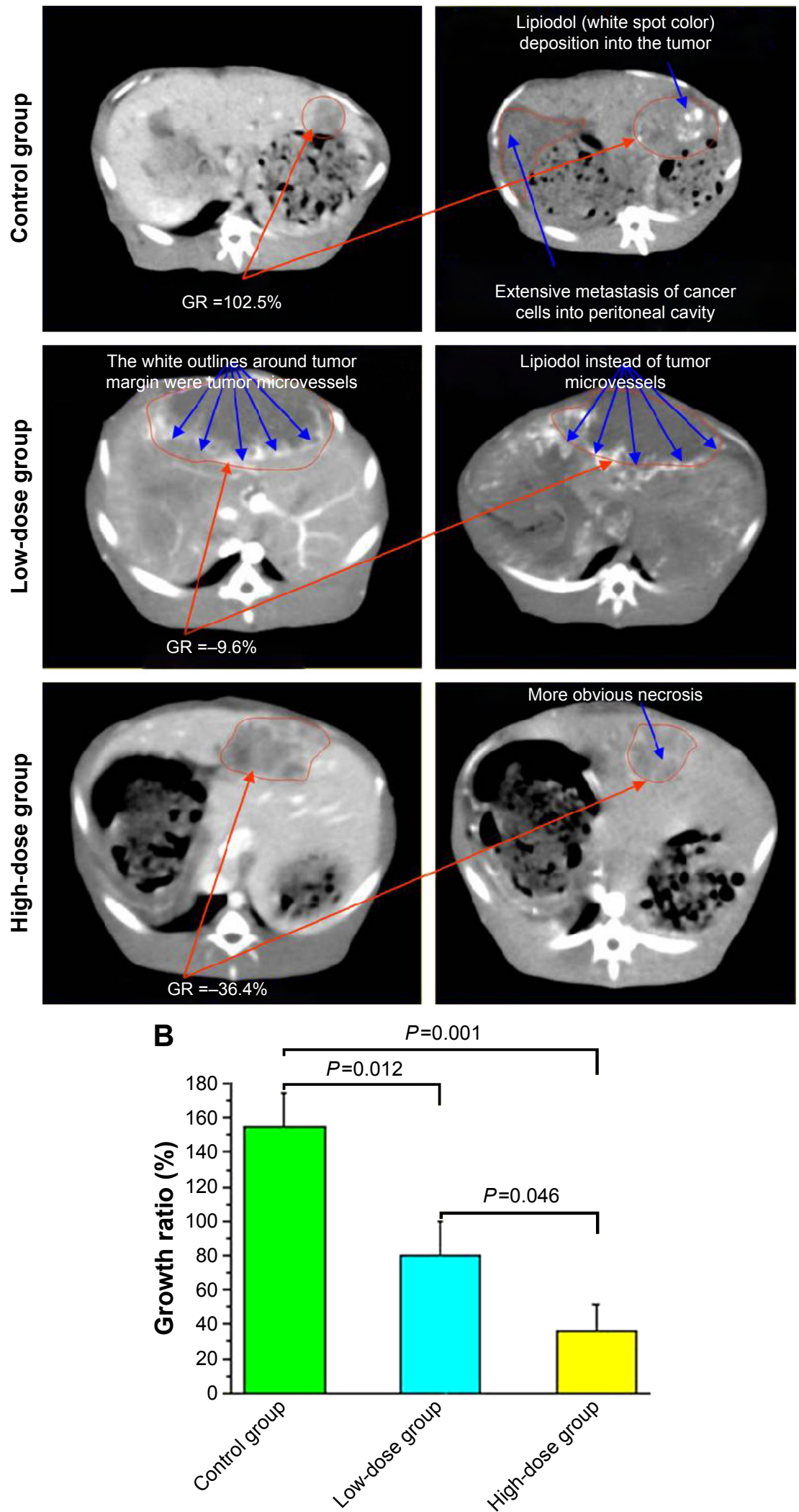

Figure 4 Effect of VEGF siRNA combined with lipiodol (TAE operation) on the growth ratios (GRs) of liver tumors induced by inoculation of VX2 tumor into the liver of rabbits by contrast-enhanced $\mathrm{CT}$ image.

Notes: (A) Representative CT images showed the liver tumor size (red circle), lipiodol deposition, and obvious necrosis. (B) Statistical analysis of tumors growth ratios in control group (ten rabbits), low-dose group (ten rabbits), and high-dose group (ten rabbits). The error bars were standard error mean of tumors growth ratios among ten rabbits in each group. Significant differences among three groups.

Abbreviations: TAE, transarterial embolization; VEGF, vascular endothelial growth factor; siRNA, small interfering RNA; CT, computed tomography. 
A 28 days after TAE operation

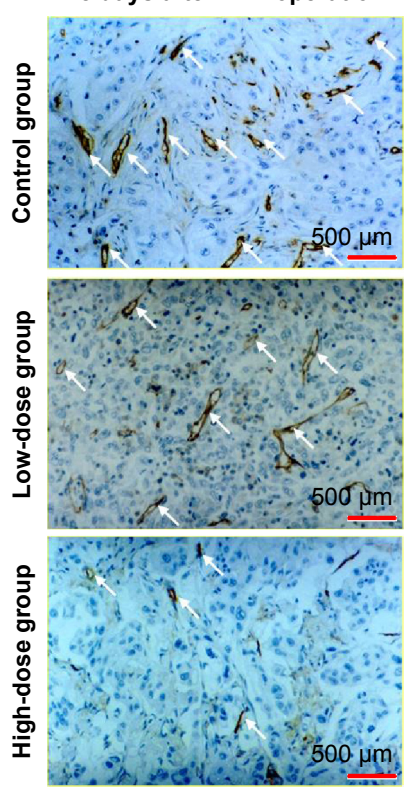

B

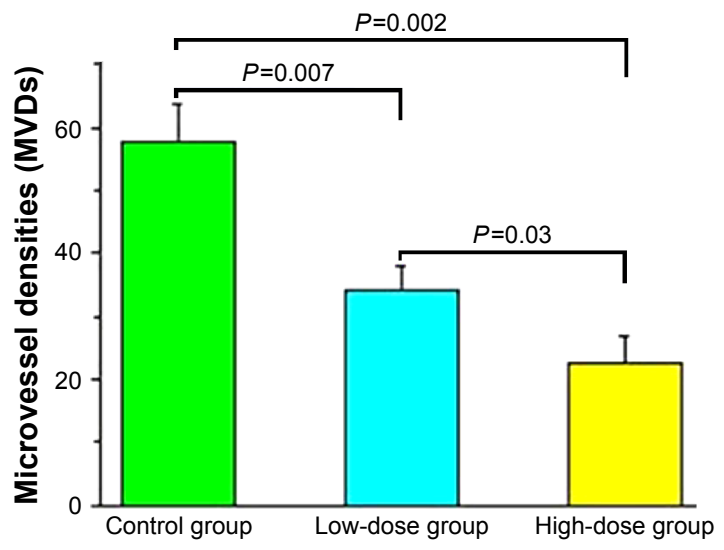

Figure 5 Effect of VEGF siRNA combined with lipiodol (TAE operation) on the microvessel densities (MVDs) of liver tumors induced by inoculation of VX2 tumor into liver of rabbits by immunohistochemical staining.

Notes: (A) The anti-CD34 monoclonal antibody was used to detect the microvessel densities (white arrows in each group). (B) Statistical analysis of MVDs in control group (ten rabbits), low-dose group (ten rabbits), and high-dose group (ten rabbits). The error bars were standard error mean of MVDs among ten rabbits in each group. Significant differences among three groups.

Abbreviations: VEGF, vascular endothelial growth factor; siRNA, small interfering RNA; TAE, transarterial embolization.
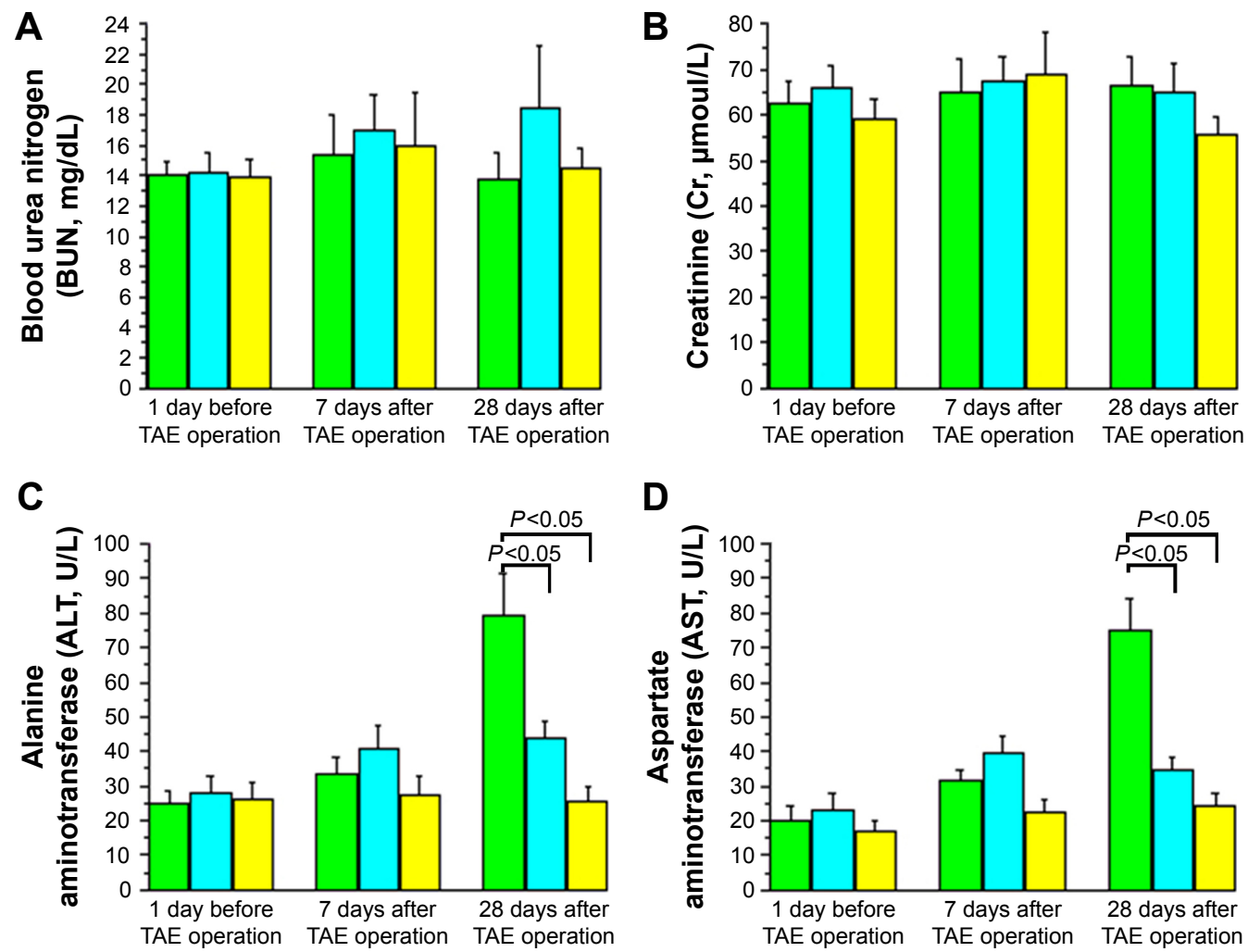

D

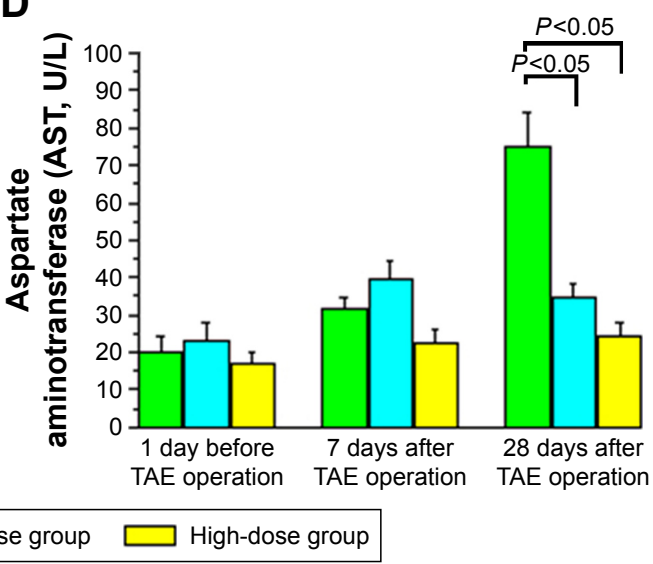

Figure 6 Toxicity evaluation of VEGF siRNA plus lipiodol (TAE operation) in livers and kidneys of rabbits.

Notes: (A) Blood urea nitrogen (BUN), (B) creatinine (Cr), (C) alanine aminotransferase (ALT), and (D) aspartate aminotransferase (AST) of serum in rabbits transplantated with VX2 tumor into the liver. Significantly different from the control group.

Abbreviations: VEGF, vascular endothelial growth factor; siRNA, small interfering RNA; TAE, transarterial embolization. 
Interventional treatment of $\mathrm{HCC}$, such as TAE, alone or combined with chemotherapy (TACE), remains a controversial treatment approach for patients with HCC. ${ }^{51,52}$ TAE or TACE usually mixed with lipiodol results in extensive tumor necrosis caused by ischemia based on the fact that the tumor blood supply depends mainly on the hepatic artery. ${ }^{53}$ Radiolabeled lipiodol has been used for targeting liver cancer and embolization of liver microvessels. ${ }^{54}$ These results clearly showed that lipiodol could accumulate around the microvessels of liver tumor in rabbit VX2 liver-transplanted model established as mentioned in a previous report, ${ }^{55}$ indicating that lipiodol has the ability to target liver cancer as mentioned in a previous report. ${ }^{54}$ Additionally, VEGF siRNA delivered by TAE technology in this study could effectively inhibit the growth of liver VX2 tumor via CT examination and the quantities of MVDs via detecting CD34 expression, indicating that targeting VEGF may be a potential treatment for liver cancer and that TAE technology may become a potential way to deliver siRNAs in clinical treatment. Furthermore, targeting VEGF via TAE technology combined with chemotherapy may become a powerful new way to effectively eliminate the insufficiency of clinical treatment of patients with HCC via TACE mentioned in "Introduction" section.

Kidney is the main organ by which drug and their metabolites are eliminated from the body and is the target for drug toxicity. The BUN and serum $\mathrm{Cr}$ are used to evaluate the functional capacity of the kidney. ${ }^{56}$ In this study, both the inoculation of VX2 tumor into the liver itself and the treatment of VX2 tumor via TAE-mediated VEGF siRNA had no toxicity to the kidneys of rabbits. ALT and AST are the important evaluation indexes of liver functions. ${ }^{57}$ Moreover, these results showed that inoculation of VX2 tumor into the liver itself at the later stage could induce significant increase in ALT and AST, indicating an obvious damage of liver functions, while VEGF siRNA delivered by TAE technology could effectively improve this kind of damage, indicating that VEGF siRNA delivered by TAE technology not only lacks toxicity to liver but also has the ability to protect liver damage induced by tumor growth.

Although TAE technology to deliver siRNAs is an effective approach used in this study for the first time, there are still some limitations needed to be investigated in further study, such as tests of stability of the siRNAs mixed with lipiodol and transfection reagent. In addition, VX2 is derived from a virus-induced papilloma, whose biological features such as growth and metastasis are similar with HCC but the sensitivity to chemotherapeutic drugs is different from HCC. Thus, in further study, we will design experiments to investigate the effect of TAE-mediated VEGF siRNA on the growth of human HCC cell models.

\section{Conclusion}

In conclusion, we have demonstrated that treatment with VEGF siRNAs could reduce the expression levels of VEGF mRNA and protein in vitro and in vivo. Additionally, we also demonstrated for the first time that targeting VEGF could inhibit the growth of VX2 cells and VX2 tumor model, as well as could decrease the MVDs of VX2 tumor in rabbit by TAE-mediated siRNA delivery technology without toxicity to liver and kidney functions, indicating that targeting VEGF via TAE-mediated siRNA delivery technology may become a powerful new option to effectively treat $\mathrm{HCC}$ in the clinic.

\section{Acknowledgments}

This project was supported by Scientific Research Fund of Zhejiang Provincial Education Department (no Y201225858) and Natural Science Foundation of Zhejiang Province (no Y2110172). The funders had no role in the study design, data collection and analysis, decision to publish, or preparation of the manuscript.

\section{Author contributions}

Zou Y, Fu C-Y, and Zhang M-M initiated the study, performed experiments, analyzed the data, and drafted the manuscript. Guo C-G, Yang Z-G, and Sun J-H conducted the in vitro analyses. Zou $\mathrm{Y}$ and $\mathrm{Fu} \mathrm{C}-\mathrm{Y}$ critically revised the manuscript. Zou Y and Fu C-Y supervised the project, advised with regard to the experimental design, and edited the manuscript. All authors contributed toward data analysis, drafting and critically revising the paper and agree to be accountable for all aspects of the work.

\section{Disclosure}

The authors report no conflicts of interest in this work.

\section{References}

1. Gomaa AI, Waked I. Recent advances in multidisciplinary management of hepatocellular carcinoma. World J Hepatol. 2015;7(4):673-687.

2. Callegari E, Gramantieri L, Domenicali M, D'Abundo L, Sabbioni S, Negrini M. MicroRNAs in liver cancer: a model for investigating pathogenesis and novel therapeutic approaches. Cell Death Differ. 2015;22(1):46-57.

3. Colagrande S, Regini F, Taliani GG, Nardi C, Inghilesi AL. Advanced hepatocellular carcinoma and sorafenib: diagnosis, indications, clinical and radiological follow-up. World J Hepatol. 2015;7(8):1041-1053.

4. Kulik LM, Chokechanachaisakul A. Evaluation and management of hepatocellular carcinoma. Clin Liver Dis. 2015;19(1):23-43.

5. Bredt LC, Mierzwa TC, Rachid AF, Fernandes FG, Zanini JC. Surgery for early stage hepatocellular carcinoma. Int J Hepatobiliary Pancreat Dis. 2015;5(1):47-55. 
6. Wang P, Song W, Li H, et al. Association between donor and recipient smoothened gene polymorphisms and the risk of hepatocellular carcinoma recurrence following orthotopic liver transplantation in a Han Chinese population. Tumour Biol. 2015;36(10):7807-7815.

7. Madkhali AA, Fadel ZT, Aljiffry MM, Hassanain MM. Surgical treatment for hepatocellular carcinoma. Saudi J Gastroenterol. 2015; 21(1):11-17.

8. Bruix J, Sherman M. Management of hepatocellular carcinoma. Hepatology. 2005;42(5):1208-1236.

9. Lo CM, Ngan H, Tso WK, et al. Randomized controlled trial of transarterial lipiodol chemoembolization for unresectable hepatocellular carcinoma. Hepatology. 2002;35(5):1164-1171.

10. Poon RT, Fan ST, Lo CM, Liu CL, Wong J. Intrahepatic recurrence after curative resection of hepatocellular carcinoma: long-term results of treatment and prognostic factors. Ann Surg. 1999;229(2):216-222.

11. Moens S, Goveia J, Stapor PC, Cantelmo AR, Carmeliet P. The multifaceted activity of VEGF in angiogenesis - Implications for therapy responses. Cytokine Growth Factor Rev. 2014;25(4):473-482.

12. Cammà C, Schepis F, Orlando A, et al. Transarterial chemoembolization for unresectable hepatocellular carcinoma: meta-analysis of randomized controlled trials. Radiology. 2002;224(1):47-54.

13. Wu HP, Feng GS, Liang HM, Zheng CS, Li X. Vascular endothelial growth factor antisense oligodeoxynucleotides with lipiodol in arterial embolization of liver cancer in rats. World J Gastroenterol. 2004;10(6):813-818.

14. Muto J, Shirabe K, Sugimachi K, Maehara Y. Review of angiogenesis in hepatocellular carcinoma. Hepatol Res. 2015;45(1):1-9.

15. Sergio A, Cristofori C, Cardin R, et al. Transcatheter arterial chemoembolization (TACE) in hepatocellular carcinoma (HCC): the role of angiogenesis and invasiveness. Am J Gastroenterol. 2008;103(4):914-921.

16. Carrillo de Santa Pau E, Arias FC, Caso Peláez E, et al. Prognostic significance of the expression of vascular endothelial growth factors $\mathrm{A}$, $\mathrm{B}, \mathrm{C}$, and D and their receptors $\mathrm{R} 1, \mathrm{R} 2$, and $\mathrm{R} 3$ in patients with nonsmall cell lung cancer. Cancer. 2009;115(8):1701-1712.

17. Mohammed RA, Green A, El-Shikh S, Paish EC, Ellis IO, Martin SG Prognostic significance of vascular endothelial cell growth factors-A, -C and -D in breast cancer and their relationship with angio- and lymphangiogenesis. Br J Cancer. 2007;96(7):1092-1100.

18. Mylona E, Alexandrou P, Mpakali A, et al. Clinicopathological and prognostic significance of vascular endothelial growth factors (VEGF)-C and -D and VEGF receptor 3 in invasive breast carcinoma. Eur J Surg Oncol. 2007;33(3):294-300.

19. Ribas C, Colleoni GW, Silva MR, Carregoza MJ, Bordin JO. Prognostic significance of vascular endothelial growth factor immunoexpression in the context of adverse standard prognostic factors in multiple myeloma Eur J Haematol. 2004;73(5):311-317.

20. Ferrara N. VEGF and the quest for tumour angiogenesis factors. Nat Rev Cancer. 2002;2(10):795-803.

21. Kupesic S, Kurjak A. Contrast-enhanced, three-dimensional power Doppler sonography for differentiation of adnexal masses. Obstet Gynecol. 2000;96(3):452-458.

22. Semela D, Dufour JF. Angiogenesis and hepatocellular carcinoma. J Hepatol. 2004;41(5):864-880.

23. Bernstein E, Caudy AA, Hammond SM, Hannon GJ. Role for a bidentate ribonuclease in the initiation step of RNA interference. Nature. 2001; 409(6818):363-366.

24. Elbashir SM, Harborth J, Lendeckel W, Yalcin A, Weber K, Tuschl T. Duplexes of 21-nucleotide RNAs mediate RNA interference in cultured mammalian cells. Nature. 2001;411(6836):494-498.

25. Teplova M, Farazi TA, Tuschl T, Patel DJ. Structural basis underlying CAC RNA recognition by the RRM domain of dimeric RNA-binding protein RBPMS. Q Rev Biophys. 2015;8:1-11.

26. Liu K, Chen H, You Q, Shi H, Wang Z. The siRNA cocktail targeting VEGF and HER2 inhibition on the proliferation and induced apoptosis of gastric cancer cell. Mol Cell Biochem. 2014;386(1-2):117-124.

27. Sun P, Yu H, Zhang WQ, Hu M, Lv R. Lentivirus-mediated siRNA targeting VEGF inhibits gastric cancer growth in vivo. Oncol Rep. 2012; 28(5):1687-1692.
28. Tao J, Tu YT, Huang CZ, et al. Inhibiting the growth of malignant melanoma by blocking the expression of vascular endothelial growth factor using an RNA interference approach. Br J Dermatol. 2005; 153(4):715-724

29. Raskopf E, Vogt A, Sauerbruch T, Schmitz V. siRNA targeting VEGF inhibits hepatocellular carcinoma growth and tumor angiogenesis in vivo. J Hepatol. 2008;49(6):977-984.

30. Medarova Z, Pham W, Farrar C, Petkova V, Moore A. In vivo imaging of siRNA delivery and silencing in tumors. Nat Med. 2007;13(3): 372-377.

31. Takeshita F, Hokaiwado N, Honma K, Banas A, Ochiya T. Local and systemic delivery of siRNAs for oligonucleotide therapy. Methods $\mathrm{Mol}$ Biol. 2009;487:83-92.

32. Gonzalez-Gonzalez E, Ra H, Hickerson RP, et al. siRNA silencing of keratinocyte-specific GFP expression in a transgenic mouse skin model. Gene Ther. 2009;16(8):963-972.

33. Song HJ, Cheng JY, Hu SL, Zhang GY, Fu Y, Zhang YJ. Value of $18 \mathrm{~F}-\mathrm{FDG} \mathrm{PET} / \mathrm{CT}$ in detecting viable tumour and predicting prognosis of hepatocellular carcinoma after TACE. Clin Radiol. 2015;70(2): 128-137.

34. Jin W, Han H, Zhou S, Wang Y, Dong T, Zhao C. Therapeutic efficacy of brucea javanica oil emulsion (BJOE) combined with transcatheter hepatic arterial chemoembolization (TACE) in patients with primary liver cancer. Int J Clin Exp Med. 2015;8(10):18954-18962.

35. Marshall WS, Kaiser RJ. Recent advances in the high-speed solid phase synthesis of RNA. Curr Opin Chem Biol. 2004;8(3):222-229.

36. Fu CY, Xia RL, Zhang TF, et al. Hemokinin-1 (4-11)-induced analgesia selectively up-regulates $\delta$-opioid receptor expression in mice. PLoS One. 2014;9(2):e90446.

37. Xia RL, Fu CY, Zhang SF, Jin YT, Zhao FK. Study on the distribution sites and the molecular mechanism of analgesia after intracerebroventricular injection of rat/mouse hemokinin-1 in mice. Peptides. 2013;43: 113-120.

38. Zhu LN, Fu CY, Zhang SF, Chen W, Jin YT, Zhao FK. Novel cytotoxic exhibition mode of antimicrobial peptide anoplin in MEL cells, the cell line of murine Friend leukemia virus - induced leukemic cells. J Pept Sci. 2013;19(9):566-574.

39. Okada M, Kudo S, Miyazaki O, et al. Antitumoral efficacy and pharmacokinetic properties of pirarubicin upon hepatic intra-arterial injection in the rabbit VX 2 tumour model. Br J Cancer. 1995;71(3): 518-524.

40. Yoon CJ, Chung JW, Park JH, et al. Transcatheter arterial chemoembolization with paclitaxel-lipiodol solution in rabbit VX2 liver tumor. Radiology. 2003;229(1):126-131.

41. Zou Y, Zhang M, Wang Q, Shang D, Wang L, Yu G. Quantitative investigation of solitary pulmonary nodules: dynamic contrast-enhanced MRI and histopathologic analysis. AJR Am J Roentgenol. 2008; 191(1):252-259.

42. Li SD, Chen YC, Hackett MJ, Huang L. Tumor-targeted delivery of siRNA by self-assembled nanoparticles. Mol Ther. 2008;16(1):163-169.

43. Schulze-Bergkamen H, Fleischer B, Schuchmann M, et al. Suppression of Mcl-1 via RNA interference sensitizes human hepatocellular carcinoma cells towards apoptosis induction. BMC Cancer. 2006;6:232.

44. Ribatti D, Vacca A, Nico B, Sansonno D, Dammacco F. Angiogenesis and anti-angiogenesis in hepatocellular carcinoma. Cancer Treat Rev. 2006;32(6):437-444.

45. Dorsett Y, Tuschl T. siRNAs: applications in functional genomics and potential as therapeutics. Nat Rev Drug Discov. 2004;3(4):318-329.

46. Yan CQ, Zhao YP. The significance of VEGF siRNA and bFGF siRNA in invasion and proliferation of pancreatic carcinoma cell. Zhonghua Wai Ke Za Zhi. 2010;48(8):610-614.

47. Lee TH, Seng S, Sekine M, et al. Vascular endothelial growth factor mediates intracrine survival in human breast carcinoma cells through internally expressed VEGFR1/FLT1. PLoS Med. 2007;4(6):e186

48. Deezagi A, Ansari-Majd S, Vaseli-Hagh N. Induced apoptosis in human prostate cancer cells by blocking of vascular endothelial growth factor by siRNA. Clin Transl Oncol. 2012;14(10):791-799. 
49. Briane D, Slimani H, Tagounits A, et al. Inhibition of VEGF expression in A431 and MDA-MB-231 tumour cells by cationic lipid-mediated siRNA delivery. J Drug Target. 2012;20(4):347-354.

50. Barr MP, Gray SG, Gately K, et al. Vascular endothelial growth factor is an autocrine growth factor, signaling through neuropilin-1 in nonsmall cell lung cancer. Mol Cancer. 2015;14:45.

51. Bruix J. Treatment of hepatocellular carcinoma. Hepatology. 1997; 25(2):259-262.

52. Trinchet JC, Beaugrand M. Treatment of hepatocellular carcinoma in patients with cirrhosis. J Hepatol. 1997;27(4):756-765.

53. Bruix J, Llovet JM, Castells A, et al. Transarterial embolization versus symptomatic treatment in patients with advanced hepatocellular carcinoma: results of a randomized, controlled trial in a single institution. Hepatology. 1998;27(6):1578-1583.
54. Jeong JM, Kim YJ, Lee YS, et al. Lipiodol solution of a lipophilic agent, 188 Re-TDD, for the treatment of liver cancer. Nucl Med Biol. 2001; 28(2):197-204.

55. Geschwind JF, Artemov D, Abraham S, et al. Chemoembolization of liver tumor in a rabbit model: assessment of tumor cell death with diffusion-weighted MR imaging and histologic analysis. $J$ Vasc Interv Radiol. 2000;11(10):1245-1255.

56. Simundic AM. Education in clinical chemistry and laboratory medicine in various European countries. Biochem Med (Zagreb). 2011;21(1):6.

57. Limdi JK, Hyde GM. Evaluation of abnormal liver function tests. Postgrad Med J. 2003;79(932):307-312. 


\section{Supplementary material}

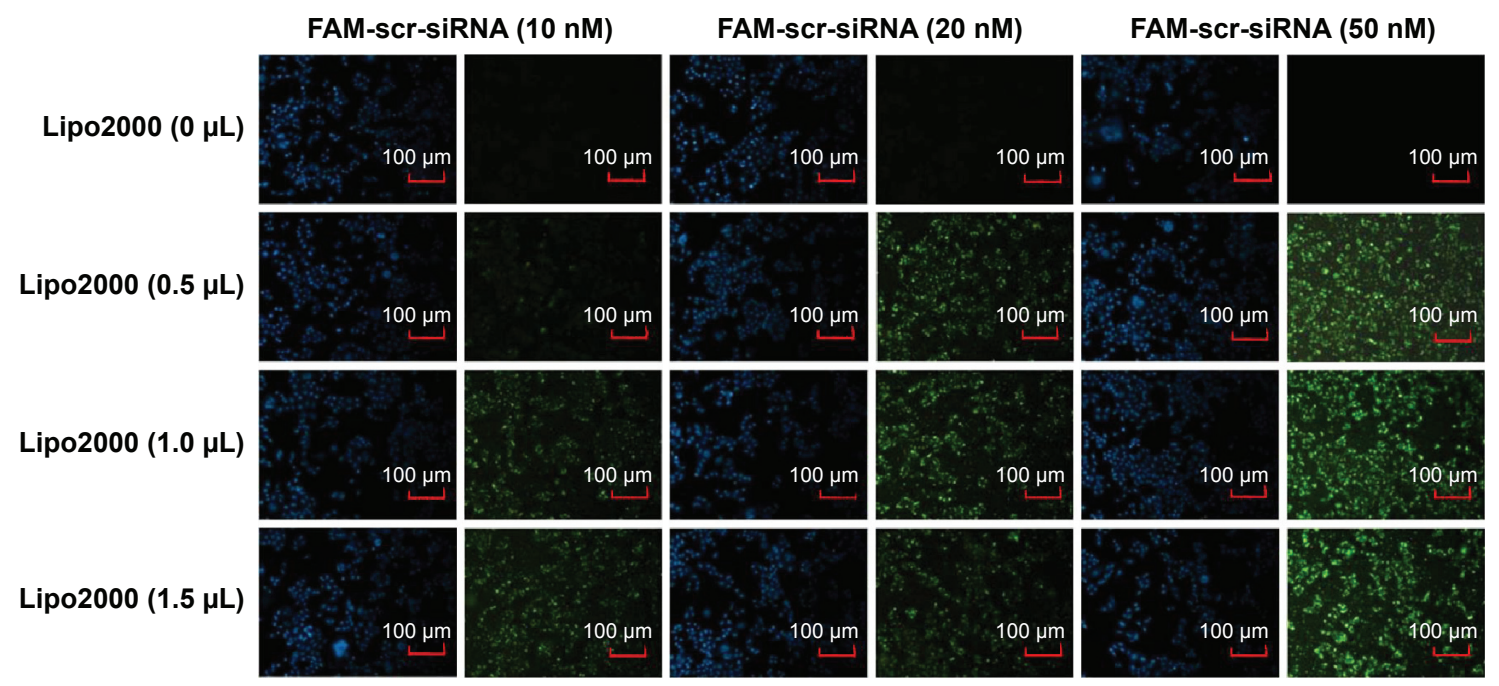

Figure SI Optimization experiment of transfection efficiency for siRNA using FAM-scr-siRNA.

Notes: VX2 cells were transduced in a 12 -well format with FAM-scr-siRNA at the concentrations of $10 \mathrm{nM}, 20 \mathrm{nM}$, and $50 \mathrm{nM}$ for 48 hours. Blue fluorescence of DAPI and green fluorescence of FAM-scr-siRNA are shown.

Abbreviations: siRNA, small interfering RNA; DAPI, 4',6-diamidino-2-phenylindole.

\section{Publish your work in this journal}

Drug Design, Development and Therapy is an international, peerreviewed open-access journal that spans the spectrum of drug design and development through to clinical applications. Clinical outcomes, patient safety, and programs for the development and effective, safe, and sustained use of medicines are a feature of the journal, which has also been accepted for indexing on PubMed Central. The manuscript management system is completely online and includes a very quick and fair peer-review system, which is all easy to use. Visit http://www.dovepress.com/testimonials.php to read real quotes from published authors.

Submit your manuscript here: http://www.dovepress.com/drug-design-development-and-therapy-journal 